\title{
Avaliação do Conhecimento de Médicos Professores, Residentes e Estudantes de Medicina acerca da Declaração de Óbito
}

\author{
Evaluation of the Knowledge of Medical \\ Professors, Residents, and Medical \\ Students regarding Death Certificate
}

Pedro Henrique Alcântara da Silva ${ }^{I}$ Amanda Samara Davi de Lima Ana Cláudia Moraes Medeiros

Beatriz Moraes Bento ${ }^{I}$ Rômulo Jerônimo Souza da Silval Fernanda Dionisio Freire ${ }^{I}$

Kleyton César Lima de Morais ${ }^{I}$ Teolinda Judite Gomes Frederico ${ }^{I}$ Lucianna Pereira da Motta Pires Correia ${ }^{I I}$

Maria do Carmo Lopes de Melo ${ }^{\text {III }}$

\section{PALAVRAS-CHAVE}

- Declaração de Óbito;

- Estudantes de Medicina;

- Educação Médica.

\section{KEYWORDS}

- Death Certificate;

- Medical Students;

- Medical Education.

\section{Recebido em: 16/06/2014}

Aprovado em: 11/04/2016

REVISTA BRASILEIRA DE EDUCAÇÃO MÉDICA

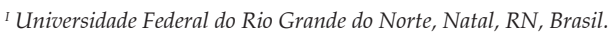

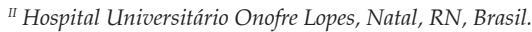

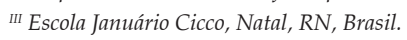




\section{INTRODUÇÃO}

A Declaração de Óbito (DO) é o documento-base do Sistema de Informações sobre Mortalidade (SIM) do Ministério da Saúde (MS) ${ }^{1}$. Seu preenchimento é um ato médico, com responsabilidade ímpar para com a saúde pública e com o avanço da ciência². Portanto, é imprescindível que as informações fornecidas nela sejam fidedignas, tempestivas e acessíveis ${ }^{1}$.

No entanto, de maneira geral, a DO é vista pelo médico apenas como uma exigência legal para o sepultamento, raramente encarada como fonte geradora de dados sobre a saúde de uma população, o que pode culminar em mau preenchimento e gerar dúvidas em relação às estatísticas de mortalidade publicadas pelo $\mathrm{MS}^{3}$.

Na maioria das capitais brasileiras, a qualidade da informação é alta e aceitável, enquanto em grande parte dos municípios não capitais é regular ou inadequada, revelando o fator socioeconômico de que, quanto mais pobres os locais onde se encontram os serviços de saúde, maior a tendência de registros insatisfatórios ${ }^{4}$.

A precariedade do preenchimento pode decorrer de vários fatores, como prontuários incompletos ou registros inadequados que não possibilitam à equipe médica uma correta ordenação dos fatos ${ }^{5}$. Além disso, muitos médicos não recebem treinamento formal sobre o preenchimento correto da DO, uma vez que este assunto é raramente abordado em escolas médicas, em hospitais e em programas de residência médica ${ }^{6}$.

Dúvidas frequentes ocorrem quanto ao momento da sua emissão; quanto ao médico que deve preenchê-la - o que encaminhou o paciente ao hospital de referência; o médico da ambulância; ou o médico que recebeu o paciente no centro de referência; e quanto ao destino de encaminhamento - o Serviço de Verificação de Óbitos (SVO) ou o Instituto Médico Legal (IML) 5, .

Tais dúvidas refletem a falta de preparo da equipe médica com relação ao assunto. Assim, são necessários mais estudos que verifiquem o real conhecimento de médicos acerca de situações de emissão desse documento, uma vez que há poucos estudos na literatura com esse enfoque.

Dessa forma, a presente pesquisa teve por objetivo avaliar em alunos matriculados no $12^{\circ}$ período do curso de Medicina da Universidade Federal do Rio Grande do Norte (UFRN), em médicos residentes e em médicos professores do Complexo Hospitalar da UFRN (CH-UFRN) o nível de conhecimento sobre os conceitos básicos inerentes ao preenchimento de uma DO e sobre questões de ordem prática acerca de sua emissão.

\section{METODOLOGIA}

O presente estudo foi realizado no $\mathrm{CH}-\mathrm{UFRN}$, composto pelo Hospital Universitário Onofre Lopes (Huol), Maternidade Es- cola Januário Cicco (MEJC) e Hospital de Pediatria (Hosped), situado no município de Natal (RN).

Trata-se de um estudo transversal com abordagem quantitativa, realizado no período de fevereiro de 2013 a junho de 2013. Foram incluídos no estudo alunos regularmente matriculados no curso de Medicina da UFRN em atividade no $12^{\circ}$ período do internato médico, último semestre do curso, bem como médicos residentes e médicos docentes do CH-UFRN que se propuseram a participar do estudo no período citado. Foram excluídos os alunos do $12^{\circ}$ período que não tinham matrícula regularizada durante a realização da pesquisa e discentes que estavam em períodos diferentes daquele fixado nos critérios de inclusão.

Pretendeu-se alcançar a totalidade, de acordo com os critérios de inclusão, dos 50 alunos de Medicina, 124 médicos residentes e 180 médicos docentes do CH-UFRN. A abordagem dos participantes foi feita de forma aleatória e não probabilística, por meio de busca ativa. Participaram da pesquisa $90 \%$ dos alunos do $12^{\circ}$ período, $53,23 \%$ dos médicos residentes e 53,34\% dos professores médicos. Para uma significância estatística de $5 \%$, objetivou-se uma amostra de 183 indivíduos. Fazendo alocação representativamente para as três categorias, foram retirados por sorteio os números de questionários excedentes, sendo arrolados 25 discentes, 64 médicos residentes e 83 médicos docentes. As perdas se justificam por não encontro do profissional, dificuldades de acesso ao mesmo e recusa na participação.

O questionário continha 11 questões de múltipla escolha, havendo apenas uma alternativa correta em cada questão. Foi construído de acordo com o Manual de Preenchimento de Declaração de Óbito do Ministério da Saúde, com base na seção de casos com perguntas e respostas da terceira edição, publicado em 2001, e também na quarta edição, publicada em 2010, complementar à terceira edição. As quatro primeiras questões trataram de conceitos básicos, e as demais abordaram aplicações em situações práticas no preenchimento da DO. O questionário foi reeditado para 11 questões após teste piloto com 40 estudantes do quinto período do curso de Medicina da UFRN.

A aplicação do questionário foi feita de forma individual e sem consulta a outros materiais de estudo ou equipamentos que possibilitassem a busca da informação. Não foi esclarecida nenhuma dúvida quanto à interpretação dos enunciados e das alternativas, pois o estudo considerou a interpretação como pertencente à avaliação e inerente à natureza do próprio instrumento utilizado, evitando-se, assim, qualquer interferência dos pesquisadores.

Os dados foram armazenados em planilha do Excel 2010, usando-se tabelas com as seguintes variáveis: porcentagem de acertos, especialidade e tempo de formação. Foram, então, realizadas comparações múltiplas, utilizando-se os testes de 
Kruskal-Wallis e de Correlação de Pearson. Os dados foram analisados e comparados no Sistema R 3.0 e no SPSS 17.0. Aplicamos essa análise de porcentagem de acertos em três categorias: estudantes de Medicina, médicos residentes e médicos professores. Calculamos a média e o desvio padrão da porcentagem de acertos em cada tabela em que se analisasse diretamente essa variável. Foi considerada nos resultados uma significância estatística com $\mathrm{p}<0,05$.

O projeto de pesquisa foi aprovado pelo Comitê de Ética do Hospital Universitário Onofre Lopes da UFRN (CEP-Huol) sob o parecer de número 39073; data da relatório: 15/06/2012.

\section{RESULTADOS}

Ao se analisarem os questionários selecionados, observa-se que a categoria que obteve a melhor média de acertos foi a dos residentes $(75,35)$, em detrimento da categoria dos estudantes $(62,88)$, que obteve a média menos satisfatória. Os professores apresentaram desempenho inferior ao dos residentes e muito semelhante ao do grupo composto por estudantes de Medicina (Tabela 1).

\begin{tabular}{|lcc}
\multicolumn{3}{c}{ TABELA 1 } \\
& Média de acertos por categorias \\
\hline \multicolumn{1}{c}{ Categoria } & Média & Desvio padrão \\
\hline Geral & 68,26 & $\pm 17,23$ \\
\hline Estudante & 62,88 & $\pm 17,56$ \\
\hline Professor & 64,61 & $\pm 17,45$ \\
\hline Residente & 75,35 & $\pm 14,51$ \\
\hline
\end{tabular}

Fonte: Dados coletados no estudo (CH-UFRN Natal/2013)

As questões presentes no instrumento de coleta foram agrupadas de acordo com o conhecimento verificado em cada item, sendo atribuídos graus de relevância aos assuntos abordados para cada grupo de questões (Tabela 2).

\begin{tabular}{ccccccc}
\multicolumn{7}{c}{ TA BELA 2 } \\
Acertos por grupos de questões x Categorias \\
\cline { 2 - 7 } Grupo & \multicolumn{7}{c|}{ Categoria } \\
\cline { 2 - 7 } & \multicolumn{7}{c}{ Estudante } & \multicolumn{2}{c|}{ Professor } & \multicolumn{2}{c}{ Residente } \\
\cline { 2 - 7 } & Média & DP & Média & DP & Média & DP \\
\hline 1 & 35,42 & $\pm 31,20$ & 44,09 & $\pm 38,20$ & 48,48 & $\pm 35,05$ \\
\hline 2 & 87,5 & $\pm 27,47$ & 84,23 & $\pm 22,30$ & 94,44 & $\pm 12,52$ \\
\hline 3 & 65,83 & $\pm 23,20$ & 65,59 & $\pm 24,74$ & 77,88 & $\pm 18,61$ \\
\hline 4 & 29,17 & $\pm 46,43$ & 41,93 & $\pm 49,61$ & 59,09 & $\pm 49,54$ \\
\hline
\end{tabular}

Fonte: Dados coletados no estudo (CH-UFRN Natal/2013)

No grupo 1 estavam as questões de pouca relevância para o preenchimento da declaração de óbito, uma vez que o erro nessas questões não interferiria na correta emissão da DO. A média de acertos entre as categorias estudadas (estudante, professor e residente) foi maior entre os residentes, mas uma média baixa, menor que 50,00, foi obtida em todas as categorias.

No grupo 2 estavam as questões consideradas relevantes por terem abordagem conceitual de termos presentes no segundo Manual da Declaração de Óbito (terceira edição). Para esse grupo, a média de acerto foi alta em todos as categorias, chegando a 94,44 na categoria dos residentes, configurando a maior média. A média obtida pelos professores foi inferior à dos estudantes nesse grupo.

No grupo 3 estavam as questões descritas como muito relevantes por serem de ordem prática no contexto de emissão da declaração. A categoria de maior média também foi a dos residentes $(77,88)$, ficando as duas outras categorias com uma média de acertos menor do que 70,00. As pontuações obtidas por estudantes e professores foram equivalentes.

O grupo 4 continha a questão considerada de conhecimento imprescindível: um caso clínico em que se deveria assinalar a alternativa que apontava as corretas causas do óbito (básica, intermediárias e final) a serem preenchidas no atestado, sendo o acerto nessa questão essencial para a correta emissão de uma declaração. A maior média obtida foi a dos residentes, com pontuação de 49,54. Não foi obtido o patamar de 60,00 de média nesta última questão. Agrupando-se as categorias duas a duas, observa-se diferença significativa quando comparamos a distinção entre estudantes e residentes, e entre professores e residentes. Esta diferença não foi estatisticamente relevante quando comparamos as categorias de estudante e professor (Tabela 3).

\begin{tabular}{|lccc|}
\hline \multicolumn{4}{c}{ TA BELA 3} \\
Comparação entre as categorias duas a duas. \\
\multicolumn{1}{|c}{ Comparação } & $\begin{array}{c}\text { Diferença } \\
\text { Observada }\end{array}$ & $\begin{array}{c}\text { Diferença } \\
\text { Crítica }\end{array}$ & Sob H \\
\hline Estudante X Professor & 3,910 & 29,034 & Não há diferença \\
\hline Estudante X Residente & 36,890 & 30,228 & Há diferença \\
\hline Professor X Residente & 32,980 & 20,410 & Há diferença
\end{tabular}

Fonte: Dados coletados no estudo (CH-UFRN Natal/2013). Teste de Kruskall Wallis

Relacionando a porcentagem de acertos com o tempo de formação, consideraram-se apenas os residentes e professores, únicas categorias que podem ser avaliadas desta maneira. $\mathrm{O}$ teste de correlação de Pearson mostrou, através de uma fórmula de modelo de regressão obtida com a análise dos dados dessas variáveis, uma estimativa de que, para cada ano a mais de tempo de formação, em média, a porcentagem de acerto diminui $0,485 \%$ (aproximadamente 0,5\%), com significância estatística ( $\mathrm{p}=0,001)$ (Figura 1). 
Figura 1

Teste de correlação- Porcentagem de Acertos X

Tempo de formação (Professores e residentes).

Correlação de Pearson

$-0,33$

p- valor

$<0,001$

Modelo de regressão: Fazendo $\mathrm{Y}=$ Porcentagem de acertos, tem-se: $\hat{Y}=74,991-0,485^{*}$ (Tempo de Formação)

\section{DISCUSSÃO}

O Sistema de Informação sobre Mortalidade contém informações valiosas para o planejamento e avaliação de ações de saúde nos níveis local, regional e nacional ${ }^{1}$. Uma dificuldade para a geração de dados de mortalidade confiáveis é o correto preenchimento da DO, o principal instrumento que alimenta esse sistema ${ }^{1}$. Diante disso, é necessário conhecer as situações práticas de emissão, conceitos básicos do tema e listar devidamente a cadeia de eventos patológicos no campo das possíveis causas de morte.

O questionário desta pesquisa avaliou esse conhecimento, e os resultados encontrados apontam limitações no preenchimento correto da DO, uma vez que o desempenho de médicos, residentes e estudantes não foi, de modo geral, satisfatório, principalmente em quesitos primordiais à correta emissão da declaração.

Os médicos docentes têm como importante papel estimular o conhecimento e o aprendizado permanente dos médicos em formação, orientando-os, da melhor maneira possível, a desenvolver inúmeras habilidades técnicas, entre as quais a competência de preencher corretamente uma Declaração de Óbito. Assim, é de extrema relevância que os professores primeiramente entendam a importância de saber conduzir de forma correta uma situação de óbito, desde o acolhimento aos familiares e acompanhantes, até a questão legal e técnica, que é da responsabilidade do profissional médico: encaminhar o corpo, quando necessário, ao devido local, bem como saber preencher criteriosamente todo o documento de maneira legível e adequada.

Entretanto, diante dos resultados encontrados na presente pesquisa, percebe-se que os professores da UFRN analisados não possuem conhecimentos suficientes sobre o tema, resultado aquém do que seria esperado para os que lidam diretamente com educação médica. Este é um dado preocupante, pois revela um evidente déficit na formação médica.

A carência de projetos de educação médica continuada, bem como o despreparo das escolas médicas podem facilmente explicar parte desses erros e dificuldades. Villar e Perez-
-Mendez ${ }^{7}$ mostraram aumento na porcentagem de DO preenchidas corretamente, de $28,9 \%$ para $91 \%$, após a apresentação de um seminário de 90 minutos sobre o tema. Segundo Santos et al. ${ }^{8}$, grande parte dos médicos considera importante o correto preenchimento do documento, porém avalia que não possui instrução suficiente a ponto de não cometer equívocos. Como uma das problemáticas, Mendonça et al. ${ }^{1}$ revelaram que cerca de $80 \%$ dos entrevistados afirmam que a maior dificuldade no correto preenchimento está na falta de clareza das instruções contidas no próprio documento.

Do ponto de vista de uma situação ideal de educação permanente por conta própria dos médicos, seja no cotidiano como médico atendente ou mesmo preenchendo DO, a tendência natural seria um aumento na porcentagem de acertos conforme o tempo de formação, mas ocorreu o oposto no presente estudo.

Uma pesquisa realizada em 2004 nos Estados Unidos ${ }^{9}$ com 4.800 questionários enviados randomicamente a residentes de medicina interna em programas de pós-graduação por todo o país, mostrou baixo desempenho dos participantes, sendo que apenas $23 \%$ atingiram a pontuação ótima do escore adotado na pesquisa. Tal pesquisa observou que, quanto maior a experiência prévia, maior seria a média de acertos.

Ao compararmos os resultados obtidos, verificamos que, na questão referente ao caso clínico em que se deveria assinalar a alternativa que apontava as corretas causas do óbito, o acerto médio em todas as categorias avaliadas foi consideravelmente pequeno. Nem mesmo na categoria de docentes houve melhor desempenho.

Problemas no preenchimento da causa do óbito são apontados na literatura como uma das maiores dificuldades enfrentadas por médicos no preenchimento do atestado. Um estudo exploratório sobre problemas de preenchimento da DO realizado em Minas Gerais apontou como principais dificuldades: desconhecimento médico sobre a importância do correto preenchimento dos campos do formulário, pouca utilização dos manuais técnicos de instrução fornecidos pelo MS, desconhecimento sobre a importância do detalhamento e a adequação da cadeia de eventos patológicos no campo das possíveis causas de morte ${ }^{1}$.

Essas dificuldades culminam em uso de diagnósticos não específicos como causa da morte ${ }^{10}$, como, por exemplo, o uso inadequado da expressão "falência múltipla de órgãos" como causa de óbito ${ }^{1}$. Outros estudos também discorrem sobre essa mesma temática, evidenciando que erros dessa natureza são um dos mais frequentes nas DO emitidas ${ }^{10-13}$.

Uma pesquisa realizada em Belém observou altas taxas de erros de preenchimento e identificou pelo menos um erro 
no preenchimento em $98,7 \%$ das DO. Os erros mais notáveis e importantes foram encontrados na descrição das condições e causas do óbito, com uma frequência de erro de $71,5 \%$, principalmente devido à imprecisão das informações ${ }^{8}$.

Vários trabalhos que avaliam a qualidade do preenchimento da DO vêm sendo publicados e têm apontado deficiências ${ }^{3,14-17}$. No entanto, nosso estudo foi pioneiro em relação à análise comparativa de conhecimento no assunto entre estudantes de Medicina, médicos residentes e médicos professores de um hospital universitário. O resultado mostrou-se inesperado, pois, em vez dos médicos docentes, responsáveis diretos pela educação médica, foram os médicos residentes que obtiveram melhores resultados. Em mais de 90,90\% das questões, os residentes foram os que obtiveram melhor desempenho.

Os residentes, de modo geral, se mostraram mais apto à emissão de uma declaração de óbito do que o profissional médico, o que pode ser justificado por serem os que estão na linha de frente nos cuidados com o paciente, sendo os responsáveis diretos por esse documento em um hospital universitário. Os residentes, portanto, acabam estudando o tema e lidando com a situação de preenchimento do documento com maior frequência. No entanto, esse melhor desempenho não deixa de ser um dado preocupante, visto que o médico docente é o responsável pela transmissão do conhecimento, avaliação e supervisão dos residentes e estudantes, devendo ter domínio do instrumento, que é um ato médico.

Esta realidade, verificada em nosso estudo, pode sugerir, entre outras coisas, uma falha no currículo e/ou falta de programas de educação continuada que abordem esse tema, que é de suma importância ${ }^{18,19,20}$. No âmbito da formação médica, é comum médicos se depararem pela primeira vez com um atestado de óbito no momento em que se veem na contingência real de preenchê-lo.

\section{CONCLUSÃO}

Este estudo evidencia a importância da educação continuada acerca do preenchimento de documentos médicos, entre eles a DO. O conhecimento adquirido na graduação deve ser permanentemente atualizado e aperfeiçoado a fim de evitar falhas e melhorar a qualidade das informações geradas com base nesses documentos. A DO tem importância humanitária, epidemiológica e legal, sendo de extrema importância o investimento em ações educativas voltadas aos alunos da graduação em Medicina e médicos formados, sejam docentes, residentes ou profissionais autônomos. Só assim será alcançada uma boa qualidade nos dados contidos na DO e em outros documentos médicos, e, consequentemente, nos dados disponibilizados pelo MS.

\section{REFERÊNCIAS}

1. Mendonça FM, Drumond E, Cardoso AMP. Problemas no preenchimento da Declaração de Óbito: estudo exploratório. Revista Brasileira de Estudos de População. 2010; 27:285-95.

2. Thompson GL. Completion of a death certificate--a physician's responsibility to medical science and public health. The West Virginia medical journal. 2011; 107(1):34-5.

3. Niobey FML, Cascão AM, Duchiade MP, Sabroza PC. Qualidade do preenchimento de atestados de óbitos de menores de um ano na região metropolitana do Rio de Janeiro. Revista de Saúde Pública. 1990; 24:311-8.

4. Kanso S, Romero DE, Leite IdC, Moraes ENd. Diferenciais geográficos, socioeconômicos e demográficos da qualidade da informação da causa básica de morte dos idosos no Brasil. Cadernos de Saúde Pública. 2011; 27:1323-39.

5. Mendonça EF, Goulart EMA, Machado JAD. Confiabilidade da declaração de causa básica de mortes infantis em região metropolitana do sudeste do Brasil. Revista de Saúde Pública. 1994; 28:385-91.

6. Lakkireddy DR, Gowda MS, Murray CW, Basarakodu KR, Vacek JL. Death certificate completion: How well are physicians trained and are cardiovascular causes overstated? American Journal of Medicine, The.117(7):492-8.

7. Villar J, Perez-Mendez L. Evaluating an educational intervention to improve the accuracy of death certification among trainees from various specialties. BMC health services research. 2007;7:183.

8. Silva JACd, Yamaki VN, Oliveira JPSd, Teixeira RKC, Santos FAF, Hosoume VSN. Declaração de óbito, compromisso no preenchimento: avaliação em Belém - Pará, em 2010. Revista da Associação Médica Brasileira. 2013; 59:335-40.

9. Lakkireddy DR, Gowda MS, Murray CW, Basarakodu KR, Vacek JL. Death certificate completion: how well are physicians trained and are cardiovascular causes overstated? The American journal of medicine. 2004; 117(7):492-8.

10. Cina SJ, Selby DM, Clark B. Accuracy of death certification in two tertiary care military hospitals. Military medicine. 1999; 164(12):897-9.

11. Zumwalt RE, Ritter MR. Incorrect death certification. An invitation to obfuscation. Postgraduate medicine. 1987; 81(8):245-7, 50, 53-4.

12. Pritt BS, Hardin NJ, Richmond JA, Shapiro SL. Death certification errors at an academic institution. Archives of pathology \& laboratory medicine. 2005;129(11):1476-9.

13. Nojilana B, Groenewald P, Bradshaw D, Reagon G. Quality of cause of death certification at an academic hospital in Cape Town, South Africa. South African medical journal = Suid-Afrikaanse tydskrif vir geneeskunde. 2009; 99(9):648-52. 
14. Laurenti R. Doenças respiratórias como causa de morte no município de São Paulo, SP (Brasil). Revista de Saúde Pública. 1981;15:353-63.

15. Strozzi GM, Strozzi JB, Souza MdLd, Schutel MD. Estudo de causa básica de óbitos de menores de 15 anos, ocorridos em hospital de Florianópolis, SC (Brasil), em 1982. Revista de Saúde Pública. 1985; 19:123-32.

16. Butler T, Islam M, Azad AK, Islam MR, Speelman P. Causes of death in diarrhoeal diseases after rehydration therapy: an autopsy study of 140 patients in Bangladesh. Bulletin of the World Health Organization. 1987; 65(3):317-23.

17. Fonseca LAM, Laurenti R. Qualidade da certificação médica da causa de morte em São Paulo, Brasil. Revista de Saúde Pública. 1974; 8:21-9.

18. Fajardo S, Aerts DRGdC, Bassanesi SL. Acurácia da equipe do Sistema de Informações sobre Mortalidade na seleção da causa básica do óbito em capital no Sul do Brasil. Cadernos de Saúde Pública. 2009; 25:2218-28.

19. Nobre LC, Victora CG, Barros FC, Lombardi C, Teixeira AMB, Fuchs SC. Avaliação da qualidade da informação sobre a causa básica de óbitos infantis no Rio Grande do Sul (Brasil). Revista de Saúde Pública. 1989; 23:207-13.

20. Heckmann IC, Canani LH, Sant'anna UL, Bordin R. Análise do preenchimento de declarações de óbitos em localidade do Estado do Rio Grande do Sul (Brasil), 1987. Revista de Saúde Pública. 1989;23:292-7.

\section{FINANCIAMENTO}

Esta pesquisa foi financiada com recursos próprios dos pesquisadores.

\section{CONTRIBUIÇÃO DOS AUTORES}

Ana Cláudia Moraes Medeiros

(Elaboração, Edição e Coleta de dados)
Amanda Samara Davi de Lima

(Elaboração, Edição e Coleta de dados)

Beatriz Moraes Bento

(Elaboração, Edição e Coleta de dados)

Fernanda Dionísio Freire

(Elaboração, Edição e Coleta de dados)

Kleyton César Lima de Morais

(Elaboração, Edição e Coleta de dados)

Lucianna Pereira da Motta Pires Correia

(Orientação, Edição e Coordenação da pesquisa)

Maria do Carmo Lopes de Melo

(Orientação, Edição e Coordenação da pesquisa)

Pedro Henrique Alcântara da Silva

(Elaboração, Edição e Coleta de dados)

Rômulo Jerônimo Souza da Silva

(Elaboração, Edição e Coleta de dados)

Teolinda Judite Gomes Frederico

(Elaboração, Edição e Coleta de dados)

\section{CONFLITO DE INTERESSES}

Declaro que não há conflitos de interesses entre os autores do artigo intitulado: "Avaliação do conhecimento de médicos professores, residentes e estudantes de medicina acerca da declaração de óbito submetido para apreciação na Revista Brasileira de Educação Médica - RBEM.

\section{ENDEREÇO PARA CORRESPONDÊNCIA}

Pedro Henrique Alcântara da Silva

Rua Padre Pinto, 796

Cidade Alta - Natal

CEP 59025-610 - RN

E-mail: phenriquealcantara@gmail.com 